Canadian

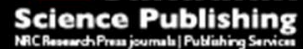

Canadian Journal of Microbiology Revue canadienne de de microbiologie

The animal gut as a melting pot for horizontal gene transfer

\begin{tabular}{|r|l|}
\hline Journal: & Canadian Journal of Microbiology \\
\hline Manuscript ID: & cjm-2015-0049.R1 \\
\hline Manuscript Type: & Mini-Review \\
\hline Date Submitted by the Author: & 14-Apr-2015 \\
\hline Complete List of Authors: & $\begin{array}{l}\text { Shterzer, Naama; Institute of Animal Science, ARO, Volcani Research } \\
\text { Center } \\
\text { Mizrahi, Itzhak; Institute of Animal Science, ARO, Volcani Research Center }\end{array}$ \\
\hline Keyword: & Horizontal gene transfer, gut microbiome, Conjugation, Plasmids, Phages \\
\hline
\end{tabular}




\section{The animal gut as a melting pot for horizontal gene transfer}

2

$3 \quad$ N. Shterzer, and I. Mizrahi ${ }^{1}$

4

5 Department of Ruminant Sciences, Institute of Animal Science, Agricultural Research

6 Organization, P.O. Box 6, Bet-Dagan 50250, Israel

7

$8{ }^{1}$ Corresponding author: I. Mizrahi; Department of Ruminant Sciences, Institute of Animal

9 Science, Agricultural Research Organization, P.O. Box 6, Bet-Dagan 50250, Israel; Tel.:

10 +972 3 9683751; Fax: +972 3 9683027; itzhakm@agri.gov.il 


\section{Abstract}

In this minireview we examine horizontal gene transfer (HGT) events in the

15 mammalian gastrointestinal tract and their role in the evolutionary adaptation of

16 microorganisms to the gut environment. We explore the notion of mammalian gut as a

17 melting pot of genetic exchange, resulting in the large extent of HGT occurrence.

18

19 Key Words: Horizontal gene transfer, gut microbiome, Conjugation, Plasmids, Phages

20 
22 Horizontal gene transfer (HGT) is a widespread phenomenon in the prokaryotic

23 world. It is commonly thought of as a key process in the evolution of bacteria and their

24 adaptation to new environments [1]. One of the most interesting microbial communities

25 resides in the mammalian gastrointestinal (GI) tract. These communities have intricate

26 relationships within them, as well as with their mammalian host $[2,3]$. The gut bacterial

27 communities are extremely diverse and complex, as well as exceptionally dense with

28 over $10^{10}$ microorganisms per milliliter of content. The immediate proximity and wide

29 range of neighboring cells create a favorable environment for HGT [4]. Here we review

30 the importance of HGT in the mammalian gut and the mechanisms by which it occurs, as

31 well as the methods available for its study.

\section{Metagenomic approaches for studying HGT}

In most studies, HGT events are identified in bacterial genomes by looking at

35 phylogenetic trees, network analysis and sequence properties such as $\mathrm{G}+\mathrm{C}$ content [5].

36 By examining bacterial genomes, one can estimate the extent of HGT and the

37 phylogenetic distances crossed by the genes. Nevertheless, a more fundamental

38 understanding of the genes that are being mobilized at a given time can be gained by

39 exploring the mobile genetic elements (MGE) themselves. These include plasmids,

40 viruses and other elements, such as transposons and integrons [6, 7]. Recent

41 developments in sequencing technologies have allowed investigators to look at overall

42 bacterial communities and describe them. These techniques can also be employed to

43 characterize communities of MGE and study the genes that they are carrying. 
Such methods have been developed for the analysis of plasmid metagenomes [8-

45 11]. Brown Kav et al. (2012) [9] analyzed the rumen plasmidome (overall plasmid

46 population) and showed the effect of the environment on its plasmids and the genes they

47 carry, as contigs sequenced in that study exhibited similarity to plasmids previously

48 isolated from rumen bacteria. In addition, the plasmid contigs showed great phylogenetic

49 mosaicism, demonstrating the large phylogenetic distances crossed in such an

50 environment. Finally, the contigs were enriched in rumen-relevant functions such as

51 respiration, and carbohydrate, amino acid and protein metabolism [9].

52 Another category of MGE that has been studied using metagenomic approaches is

53 the virome - the overall virus population in the environment. Several studies have

54 attempted to characterize the mammalian GI tract virome using similar techniques [12-

55 17]. In this case, one of the main challenges encountered by investigators was the

56 extremely low similarity of the obtained sequences to known genes in the different

57 databases. Of the small number of sequences that could be identified, most were

58 associated with DNA metabolism, replication and repair [16, 17]. Other overrepresented

59 functions could not be detected, most likely due to the lack of proper database

60 representation. These studies also identified sequences of prophages, indicating that

61 transduction is one of the mechanisms by which HGT occurs in these systems [12-17].

62

63 HGT in individual genomes

64 Numerous studies have described specific HGT events in microbial genomes.

65 Meehan and Beiko (2012) [18] detected multiple events of HGT of a peptides/nickel

66 transport complex in strains of the gut microbe Faecalibacterium prausnitzii, each operon 
67 originating from a different taxonomic source, but all from other gut-inhabiting 68 Clostridiales [18]. Coyne et al. (2014) [19] found HGT events between different

69 Bacteroidales species in the gut of individual humans. These events were likely due to 70 integrative conjugative elements, and encoded fitness-amplifying functions involving 71 orphan DNA methylases, fimbriae synthesis proteins, putative secreted antimicrobial 72 molecules, and a predicted type VI secretion system [19]. An interesting case involved 73 the transfer of porphyranases and agarases from marine Bacteroidetes into the genome of 74 Bacteroides plebeius. These genes were further found in the microbiome of Japanese 75 subjects, and were missing from North American ones. As these donating bacteria are 76 associated with seaweed, this finding serves as an indication of the manner in which this 77 bacterium obtained the genes for porphyran and agar degradation: high seaweed 78 consumption in the Japanese population enabled this transfer, and most likely maintained 79 it, as the substrates of these genes are common in the Japanese gut [20].

\section{HGT over phylogenetic distances}

A wider view of the effect of HGT on gut microbial genomes was reported by

83 Zaneveld et al. (2010) [21]. These authors characterized gene content conservation with 84 regard to phylogenetic distances and environment by analyzing the gene content 85 conservation in bacterial genomes isolated from different environments and the plasmids 86 sequenced with them. Their analysis showed that gene content in gut bacterial 87 chromosomes is more conserved over long phylogenetic distances than that in non-gut 88 bacterial chromosomes. Reciprocally, gene content over short phylogenetic distances was 89 less conserved in gut vs. non-gut bacterial chromosomes. The same phenomenon was 
90 observed for the analyzed plasmids. The authors suggested that over long phylogenetic

91 distances, the distinctive functional requirements of the gut environment dictate the

92 genetic repertoire, whereas closely related bacteria in the gut are driven to specialization

93 [21]. This suggests that HGT sculpts the microbial community and the similarity among

94 its genomes in the gut environment, and somehow outweighs the constraints imposed by

95 phylogenetic distances, such as $\mathrm{G}+\mathrm{C}$ content and restriction systems $[5,22,23]$. This is

further supported by the analysis of HGT events identified in sequenced genomes. The

authors concluded that the main driver of HGT events is the ecological niche, signifying

adaptation to that niche [24].

HGT events in the mammalian GI tract have been found to bridge a range of

100 phylogenetic distances, including different domains. Evidence of inter-domain HGT was

101 found in the gut archaeon Methanobrevibacter smithii, where $15 \%$ of its genes were

102 suspected of having been transferred from co-inhabiting gut bacteria. These genes were

103 in large part adjacent to mobile genetic elements in $M$. smithii's genome, such as phage-

104 related proteins, integrases, transposases and insertion sequence elements. The newly

105 acquired genes mostly encoded surface-related proteins that contributed to the archaeon's

106 adaptation to the gut environment [25]. Furthermore, Garcia-Vallvé et al. (2000) [26]

107 suggested that many of the glycosyl hydrolases of rumen fungi had been acquired by

108 HGT from bacteria, also exemplifying cross-domain gene transfer [26]. Similarly, the

109 transfer of environment-relevant genes was discovered in rumen ciliates. Ricard et al.

110 (2006) [27] proposed that HGT enabled ciliates to inhabit the rumen by introducing

111 advantageous functions involved in the degradation of plant cell wall - a substrate that is

112 plentiful in the rumen environment [27]. 


\section{4} conjugation has a cardinal role in HGT within gut microbiomes. Therefore, a comparison

134 of these two mobile entities and their contribution to HGT in these environments could be 135 of great importance to understanding the forces driving HGT. 


\section{Conclusions}

HGT is a central mechanism for genome diversification and the acquisition of

139 novel functions. It is therefore a driver of microbial evolution and ecology. Evidence

140 suggests that in the mammalian GI tact, HGT events have allowed microbes to adapt to

141 this selective and demanding environment by conferring gut-relevant functions as well as antimicrobial drug resistance and virulence genes [34] and enabling the utilization of new niches. Plasmid conjugation in gut environments could be a favored process for HGT due 144 to the dense and diverse nature of the microbial populations that occupy them and the 145 protection it provides to the DNA during its transfer. This environment is essentially a 146 "melting pot" of genetic exchange, resulting in frequent HGT events crossing varying 147 phylogenetic distances. It is thus of major interest for the study of gut microbiomes and 148 HGT-facilitated bacterial evolution.

149 Although there are many indications of HGT in the mammalian GI tract, a 150 broader view of this evolutionarily important phenomenon is required. Recent 151 technological advances have allowed investigators to access total populations of MGE 152 and investigate HGT processes from a community perspective. While these studies 153 provide important initial information, much work is needed to understand the ecological 154 and evolutionary roles played by HGT in a complex and restrictive environment such as 155 the mammalian GI tract.

156 Understanding HGT within the mammalian GI tract will provide a better 157 understanding of the microbial population residing there, as well as of the universal 158 evolutionary processes steered by such events. 


\section{References}

Arber, W., 2014. Horizontal Gene Transfer among Bacteria and Its Role in Biological Evolution. Life, 4(2): p. 217-224.

Berg Miller, M.E., et al. 2012. Phage-bacteria relationships and CRISPR elements revealed by a metagenomic survey of the rumen microbiome. Environmental Microbiology, 14(1): p. 207-227.

Breitbart, M., et al. 2003. Metagenomic analyses of an uncultured viral community from human feces. Journal of bacteriology,. 185(20): p. 6220-6223.

Broaders, E., Gahan, C.G. and Marchesi, J.R. 2013. Mobile genetic elements of the human gastrointestinal tract: potential for spread of antibiotic resistance genes. Gut microbes, 4(4): p. 271-280.

Brown Kav, A., et al. 2012. Insights into the bovine rumen plasmidome. Proc Natl Acad Sci U S A, 109(14): p. 5452-7.

Brown Kav, A., Benhar, I. and Mizrahi, I. 2013. A method for purifying high quality and high yield plasmid DNA for metagenomic and deep sequencing approaches. Journal of microbiological methods, 95(2): p. 272-279.

Capozzi, V. and Spano G., 2009. Horizontal gene transfer in the gut: Is it a risk? Food Research International, 42(10): p. 1501-1502.

Coyne, M.J., et al. 2014 .Evidence of extensive DNA transfer between bacteroidales species within the human gut. MBio, 5(3): p. e01305-14.

Darmon, E. and Leach, D.R.F. 2014. Bacterial Genome Instability. Microbiology and Molecular Biology Reviews, 78(1): p. 1-39.

Flint, H.J. 1994. Molecular genetics of obligate anaerobes from the rumen. FEMS microbiology letters, 121(3): p. 259-267.

Flint, H.J., The rumen microbial ecosystem - some recent developments. Trends in Microbiology, 1997. 5(12): p. 483-488.

Garcia-Vallve, S., Romeu, A. and Palau, J. 2000. Horizontal gene transfer of glycosyl hydrolases of the rumen fungi. Mol Biol Evol, 17(3): p. 352-61.

Hehemann, J.-H., et al. 2010. Transfer of carbohydrate-active enzymes from marine bacteria to Japanese gut microbiota. Nature, 464(7290): p. 908-912.

Jones, B.V. and Marchesi, J.R. 2007. Transposon-aided capture (TRACA) of plasmids resident in the human gut mobile metagenome. Nat Meth, 4(1): p. 55-61. 
Jørgensen, T.S., et al. 2014. Hundreds of Circular Novel Plasmids and DNA Elements Identified in a Rat Cecum Metamobilome. PLoS ONE, 9(2): p. e87924.

Kim, M.S., et al. 2011. Diversity and abundance of single-stranded DNA viruses in human feces. Appl Environ Microbiol, 77(22): p. 8062-70.

Koonin, E.V. and Wolf, Y.I. 2008. Genomics of bacteria and archaea: the emerging dynamic view of the prokaryotic world. Nucleic acids research, 2008. 36(21): p. 6688-6719.Ley, R.E., et al., Worlds within worlds: evolution of the vertebrate gut microbiota. Nat Rev Micro, 6(10): p. 776-788.

Kurokawa, K., et al. 2007. Comparative Metagenomics Revealed Commonly Enriched Gene Sets in Human Gut Microbiomes. DNA Research, 14(4): p. 169-181.

Lurie-Weinberger, Peeri, M.N., M. and Gophna, U. 2012. Contribution of lateral gene transfer to the gene repertoire of a gut-adapted methanogen. Genomics, 99(1): p. 52-58.

McCuddin, Z.P., et al. 2006. Klebsiella to Salmonella gene transfer within rumen protozoa: implications for antibiotic resistance and rumen defaunation. Vet Microbiol, 114(3-4): $p$. 275-84.

Meehan, C.J. and Beiko, R.G. 2012. Lateral gene transfer of an ABC transporter complex between major constituents of the human gut microbiome. BMC microbiology, 12(1): p. 248.

Minot, S., et al. 2011. The human gut virome: inter-individual variation and dynamic response to diet. Genome Res, 21(10): p. 1616-25.

Moliner, C., P.E. Fournier, and Raoult, D. 2010. Genome analysis of microorganisms living in amoebae reveals a melting pot of evolution. FEMS microbiology reviews, 34(3): p. 281294.

Norman, A., Hansen, L.H. and Sørensen, S.J. 2009. Conjugative plasmids: vessels of the communal gene pool. Philosophical Transactions of the Royal Society B: Biological Sciences, 364(1527): p. 2275-2289.

Popa, O., et al. 2011. Directed networks reveal genomic barriers and DNA repair bypasses to lateral gene transfer among prokaryotes. Genome Res, 21(4): p. 599-609.

Reyes, A., et al. 2010. Viruses in the faecal microbiota of monozygotic twins and their mothers. Nature, 466(7304): p. 334-8.

Reyes, A., et al. 2012. Going viral: next-generation sequencing applied to phage populations in the human gut. Nat Rev Microbiol, 10(9): p. 607-17.

Ricard, G., et al. 2006. Horizontal gene transfer from Bacteria to rumen Ciliates indicates adaptation to their anaerobic, carbohydrates-rich environment. BMC Genomics, 7: $p$. 22. 
254 Ross, E.M., et al. 2013. Metagenomics of rumen bacteriophage from thirteen lactating dairy cattle. BMC microbiology, 13(1): p. 242.

Smillie, C.S., et al. 2011. Ecology drives a global network of gene exchange connecting the human microbiome. Nature, 480(7376): p. 241-244.

Stecher, B., et al. 2012. Gut inflammation can boost horizontal gene transfer between pathogenic and commensal Enterobacteriaceae. Proceedings of the National Academy of Sciences, 109(4): p. 1269-1274.

Thomas, C.M. and Nielsen, K.M. 2005.Mechanisms of, and Barriers to, Horizontal Gene Transfer between Bacteria. Nat Rev Micro, 3(9): p. 711-721.

Zaneveld, J.R., et al. 2010. Ribosomal RNA diversity predicts genome diversity in gut bacteria and their relatives. Nucleic Acids Research, 38(12): p. 3869-3879.

270

271 


\section{The animal gut as a melting pot for horizontal gene transfer}

2

3 N. Shterzer and I. Mizrahi ${ }^{1}$

4

5 Department of Ruminant Sciences, Institute of Animal Science, Agricultural Research

6 Organization, P.O. Box 6, Bet-Dagan 50250, Israel

7

$8{ }^{1}$ Corresponding author: I. Mizrahi; Department of Ruminant Sciences, Institute of Animal

9 Science, Agricultural Research Organization, P.O. Box 6, Bet-Dagan 50250, Israel; Tel.:

10 +972 3 9683751; Fax: +972 3 9683027; itzhakm@agri.gov.il 


\section{Abstract}

13 In this minireview we examine horizontal gene transfer (HGT) events in the 14 mammalian gastrointestinal tract and their role in the evolutionary adaptation of 15 microorganisms to the gut environment. We explore the notion of mammalian gut as a 16 melting pot of genetic exchange, resulting in the large extent of HGT occurrence.

17

18 
Horizontal gene transfer (HGT) is a widespread phenomenon in the prokaryotic

20 world. It is commonly thought of as a key process in the evolution of bacteria and their

21 adaptation to new environments (Koonin and Wolf 2008). One of the most interesting

22 microbial communities resides in the mammalian gastrointestinal (GI) tract. These

23 communities have intricate relationships within them, as well as with their mammalian

24 host (Flint 1997, Ley, Lozupone et al. 2008). The gut bacterial communities are

25 extremely diverse and complex, as well as exceptionally dense with over $10^{10}$

26 microorganisms per milliliter of content. The immediate proximity and wide range of

27 neighboring cells create a favorable environment for HGT (Flint 1994). Here we review

28 the importance of HGT in the mammalian gut and the mechanisms by which it occurs, as

29 well as the methods available for its study.

\section{Metagenomic approaches for studying HGT}

In most studies, HGT events are identified in bacterial genomes by looking at

33 phylogenetic trees, network analysis and sequence properties such as $\mathrm{G}+\mathrm{C}$ content (Popa,

34 Hazkani-Covo et al. 2011). By examining bacterial genomes, one can estimate the extent

35 of HGT and the phylogenetic distances crossed by the genes. Nevertheless, a more

36 fundamental understanding of the genes that are being mobilized at a given time can be

37 gained by exploring the mobile genetic elements (MGE) themselves. These include

38 plasmids, viruses and other elements, such as transposons and integrons (Broaders,

39 Gahan et al. 2013, Darmon and Leach 2014). Recent developments in sequencing

40 technologies have allowed investigators to look at overall bacterial communities and 
41 describe them. These techniques can also be employed to characterize communities of

42 MGE and study the genes that they are carrying.

Such methods have been developed for the analysis of plasmid metagenomes

44 (Jones and Marchesi 2007, Brown Kav, Sasson et al. 2012, Brown Kav, Benhar et al.

45 2013, Jørgensen, Xu et al. 2014). Brown Kav, Sasson et al. (2012) analyzed the rumen

46 plasmidome (overall plasmid population) and showed the effect of the environment on its

47 plasmids and the genes they carry, as contigs sequenced in that study exhibited similarity

48 to plasmids previously isolated from rumen bacteria. In addition, the plasmid contigs

49 showed great phylogenetic mosaicism, demonstrating the large phylogenetic distances

50 crossed in such an environment. Finally, the contigs were enriched in rumen-relevant

51 functions such as respiration, and carbohydrate, amino acid and protein metabolism

52 (Brown Kav, Sasson et al. 2012).

53 Another category of MGE that has been studied using metagenomic approaches is

54 the virome - the overall virus population in the environment. Several studies have

55 attempted to characterize the mammalian GI tract virome using similar techniques

56 (Breitbart, Hewson et al. 2003, Reyes, Haynes et al. 2010, Kim, Park et al. 2011, Minot,

57 Sinha et al. 2011, Berg Miller, Yeoman et al. 2012, Ross, Petrovski et al. 2013). In this

58 case, one of the main challenges encountered by investigators was the extremely low

59 similarity of the obtained sequences to known genes in the different databases. Of the

60 small number of sequences that could be identified, most were associated with DNA

61 metabolism, replication and repair (Berg Miller, Yeoman et al. 2012, Ross, Petrovski et

62 al. 2013). Other overrepresented functions could not be detected, most likely due to the

63 lack of proper database representation. These studies also identified sequences of 
64 prophages, indicating that transduction is one of the mechanisms by which HGT occurs

65 in these systems (Breitbart, Hewson et al. 2003, Reyes, Haynes et al. 2010, Kim, Park et

66 al. 2011, Minot, Sinha et al. 2011, Berg Miller, Yeoman et al. 2012, Ross, Petrovski et al.

67 2013).

68

69 HGT in individual genomes

Numerous studies have described specific HGT events in microbial genomes.

71 Meehan and Beiko (2012) detected multiple events of HGT of a peptides/nickel transport

72 complex in strains of the gut microbe Faecalibacterium prausnitzii, each operon

73 originating from a different taxonomic source, but all from other gut-inhabiting

74 Clostridiales (Meehan and Beiko 2012). Coyne, Zitomersky et al. (2014) found HGT

75 events between different Bacteroidales species in the gut of individual humans. These

76 events were likely due to integrative conjugative elements, and encoded fitness-

77 amplifying functions involving orphan DNA methylases, fimbriae synthesis proteins,

78 putative secreted antimicrobial molecules, and a predicted type VI secretion system

79 (Coyne, Zitomersky et al. 2014). An interesting case involved the transfer of

80 porphyranases and agarases from marine Bacteroidetes into the genome of Bacteroides

81 plebeius. These genes were further found in the microbiome of Japanese subjects, and

82 were missing from North American ones. As these donating bacteria are associated with

83 seaweed, this finding serves as an indication of the manner in which this bacterium

84 obtained the genes for porphyran and agar degradation: high seaweed consumption in the

85 Japanese population enabled this transfer, and most likely maintained it, as the substrates

86 of these genes are common in the Japanese gut (Hehemann, Correc et al. 2010). 
HGT over phylogenetic distances

A wider view of the effect of HGT on gut microbial genomes was reported by

90 Zaneveld, Lozupone et al. (2010). These authors characterized gene content conservation

91 with regard to phylogenetic distances and environment by analyzing the gene content

92 conservation in bacterial genomes isolated from different environments and the plasmids

93 sequenced with them. Their analysis showed that gene content in gut bacterial

94 chromosomes is more conserved over long phylogenetic distances than that in non-gut

95 bacterial chromosomes. Reciprocally, gene content over short phylogenetic distances was

96 less conserved in gut vs. non-gut bacterial chromosomes. The same phenomenon was

97 observed for the analyzed plasmids. The authors suggested that over long phylogenetic

98 distances, the distinctive functional requirements of the gut environment dictate the

99 genetic repertoire, whereas closely related bacteria in the gut are driven to specialization

100 (Zaneveld, Lozupone et al. 2010). This suggests that HGT sculpts the microbial

101 community and the similarity among its genomes in the gut environment, and somehow

102 outweighs the constraints imposed by phylogenetic distances, such as $\mathrm{G}+\mathrm{C}$ content and

103 restriction systems (Thomas and Nielsen 2005, Popa, Hazkani-Covo et al. 2011, Arber

104 2014). This is further supported by the analysis of HGT events identified in sequenced

genomes. The authors concluded that the main driver of HGT events is the ecological

niche, signifying adaptation to that niche (Smillie, Smith et al. 2011).

HGT events in the mammalian GI tract have been found to bridge a range of

108 phylogenetic distances, including different domains. Evidence of inter-domain HGT was

109 found in the gut archaeon Methanobrevibacter smithii, where $15 \%$ of its genes were 
110 suspected of having been transferred from co-inhabiting gut bacteria. These genes were

111 in large part adjacent to mobile genetic elements in $M$. smithii's genome, such as phage-

112 related proteins, integrases, transposases and insertion sequence elements. The newly

113 acquired genes mostly encoded surface-related proteins that contributed to the archaeon's

114 adaptation to the gut environment (Lurie-Weinberger, Peeri et al. 2012). Furthermore,

115 Garcia-Vallvé, Romeu et al. (2000) suggested that many of the glycosyl hydrolases of

116 rumen fungi had been acquired by HGT from bacteria, also exemplifying cross-domain

117 gene transfer (Garcia-Vallve, Romeu et al. 2000). Similarly, the transfer of environment-

118 relevant genes was discovered in rumen ciliates. Ricard, McEwan et al. (2006) proposed

119 that HGT enabled ciliates to inhabit the rumen by introducing advantageous functions

120 involved in the degradation of plant cell wall - a substrate that is plentiful in the rumen

121 environment (Ricard, McEwan et al. 2006).

122

123

Mechanisms for HGT in the mammalian GI tract

One of the main forces enabling this large extent of HGT in the gut, as well as

125 cross-domain gene transfer, could be physical proximity. This was shown in the aquatic

126 environment, where intra-amoebal microorganisms obtain many genes from nearby

127 bacteria, as well as their amoeba hosts. It was suggested that the amoebae serve as a

128 "genetic melting pot", creating optimal conditions for HGT between the bacteria residing

129 within them (Moliner, Fournier et al. 2010). Rumen ciliates have also been shown to play

130 a role in promoting HGT between bacteria in the rumen. McCuddin, Carlson et al. (2006)

131 demonstrated that gene transfer occurs, most likely via conjugation, within the ciliate

132 itself. They hypothesized that the conditions inside the ciliate-close proximity of the 
133 donor and receptor coupled with stress induced by engulfment by the ciliate-are 134 manifested in increased occurrence of HGT (McCuddin, Carlson et al. 2006).

135 Such conditions of extreme density also characterize the mammalian gut itself.

136 One of the mechanisms for HGT that can be markedly affected by physical proximity is 137 conjugation_(Kurokawa, Itoh et al. 2007, Stecher, Denzler et al. 2012). This mechanism is 138 also advantageous in the gut system since it protects the DNA from nucleases and heavy 139 metals that are present in the environment (Norman, Hansen et al. 2009). Viruses also 140 provide such protection during DNA transfer, but unlike marine environments, where 141 viruses are extremely abundant and outnumber bacteria 10:1, this ratio in the mammalian

142 GI tract has been reported to be about 1:1 (Reyes, Semenkovich et al. 2012). This 143 suggests that conjugation has a cardinal role in HGT within gut microbiomes. Therefore, 144 a comparison of these two mobile entities and their contribution to HGT in these 145 environments could be of great importance to understanding the forces driving HGT.

\section{Conclusions}

148 HGT is a central mechanism for genome diversification and the acquisition of 149 novel functions. It is therefore a driver of microbial evolution and ecology. Evidence 150 suggests that in the mammalian GI tact, HGT events have allowed microbes to adapt to 151 this selective and demanding environment by conferring gut-relevant functions as well as 152 antimicrobial drug resistance and virulence genes (Capozzi and Spano 2009) and 153 enabling the utilization of new niches. Plasmid conjugation in gut environments could be 154 a favored process for HGT due to the dense and diverse nature of the microbial 155 populations that occupy them and the protection it provides to the DNA during its 
156 transfer. This environment is essentially a "melting pot" of genetic exchange, resulting in 157 frequent HGT events crossing varying phylogenetic distances. It is thus of major interest 158 for the study of gut microbiomes and HGT-facilitated bacterial evolution.

159 Although there are many indications of HGT in the mammalian GI tract, a 160 broader view of this evolutionarily important phenomenon is required. Recent 161 technological advances have allowed investigators to access total populations of MGE 162 and investigate HGT processes from a community perspective. While these studies 163 provide important initial information, much work is needed to understand the ecological 164 and evolutionary roles played by HGT in a complex and restrictive environment such as 165 the mammalian GI tract.

166 Understanding HGT within the mammalian GI tract will provide a better 167 understanding of the microbial population residing there, as well as of the universal 168 evolutionary processes steered by such events. 


\section{References}

Arber, W., Horizontal Gene Transfer among Bacteria and Its Role in Biological Evolution. Life, 2014. 4(2): p. 217-224.Arber, W. (2014). "Horizontal Gene Transfer among Bacteria and Its Role in Biological Evolution." Life 4(2): 217-224.

Berg Miller, M. E., C. J. Yeoman, N. Chia, S. G. Tringe, F. E. Angly, R. A. Edwards, H. J. Flint, R. Lamed, E. A. Bayer and B. A. White (2012). "Phage-bacteria relationships and CRISPR elements revealed by a metagenomic survey of the rumen microbiome." Environmental Microbiology 14(1): 207-227.

Breitbart, M., I. Hewson, B. Felts, J. M. Mahaffy, J. Nulton, P. Salamon and F. Rohwer (2003). "Metagenomic analyses of an uncultured viral community from human feces." Journal of bacteriology 185(20): 6220-6223.

Broaders, E., C. G. Gahan and J. R. Marchesi (2013). "Mobile genetic elements of the human gastrointestinal tract: potential for spread of antibiotic resistance genes." Gut microbes 4(4): 271-280.

Brown Kav, A., I. Benhar and I. Mizrahi (2013). "A method for purifying high quality and high yield plasmid DNA for metagenomic and deep sequencing approaches." Journal of microbiological methods 95(2): 272-279.

Brown Kav, A., G. Sasson, E. Jami, A. Doron-Faigenboim, I. Benhar and I. Mizrahi (2012). "Insights into the bovine rumen plasmidome." Proc Natl Acad Sci U S A 109(14): 5452-5457.

Capozzi, V. and G. Spano (2009). "Horizontal gene transfer in the gut: Is it a risk?" Food Research International 42(10): 1501-1502.

Coyne, M. J., N. L. Zitomersky, A. M. McGuire, A. M. Earl and L. E. Comstock (2014). "Evidence of extensive DNA transfer between bacteroidales species within the human gut." MBio 5(3): e01305-01314.

Darmon, E. and D. R. F. Leach (2014). "Bacterial Genome Instability." Microbiology and Molecular Biology Reviews 78(1): 1-39.

Flint, H. J. (1994). "Molecular genetics of obligate anaerobes from the rumen." FEMS microbiology letters 121(3): 259-267.

Flint, H. J. (1997). "The rumen microbial ecosystem-some recent developments." Trends in Microbiology 5(12): 483-488.

Garcia-Vallve, S., A. Romeu and J. Palau (2000). "Horizontal gene transfer of glycosyl hydrolases of the rumen fungi." Mol Biol Evol 17(3): 352-361.

Hehemann, J.-H., G. Correc, T. Barbeyron, W. Helbert, M. Czjzek and G. Michel (2010). "Transfer of carbohydrate-active enzymes from marine bacteria to Japanese gut microbiota." Nature 464(7290): 908-912.

Jones, B. V. and J. R. Marchesi (2007). "Transposon-aided capture (TRACA) of plasmids resident in the human gut mobile metagenome." Nat Meth 4(1): 55-61.

Jørgensen, T. S., Z. Xu, M. A. Hansen, S. J. Sørensen and L. H. Hansen (2014). "Hundreds of Circular Novel Plasmids and DNA Elements Identified in a Rat Cecum Metamobilome." PLoS ONE 9(2): e87924.

Kim, M. S., E. J. Park, S. W. Roh and J. W. Bae (2011). "Diversity and abundance of single-stranded DNA viruses in human feces." Appl Environ Microbiol 77(22): 8062-8070. 
Koonin, E. V. and Y. I. Wolf (2008). "Genomics of bacteria and archaea: the emerging dynamic view of the prokaryotic world." Nucleic acids research 36(21): 66886719.

Kurokawa, K., T. Itoh, T. Kuwahara, K. Oshima, H. Toh, A. Toyoda, H. Takami, H. Morita, V. K. Sharma, T. P. Srivastava, T. D. Taylor, H. Noguchi, H. Mori, Y. Ogura, D. S. Ehrlich, K. Itoh, T. Takagi, Y. Sakaki, T. Hayashi and M. Hattori (2007). "Comparative Metagenomics Revealed Commonly Enriched Gene Sets in Human Gut Microbiomes." DNA Research 14(4): 169-181.

Ley, R. E., C. A. Lozupone, M. Hamady, R. Knight and J. I. Gordon (2008). "Worlds within worlds: evolution of the vertebrate gut microbiota." Nat Rev Micro 6(10): 776-788.

Lurie-Weinberger, M. N., M. Peeri and U. Gophna (2012). "Contribution of lateral gene transfer to the gene repertoire of a gut-adapted methanogen." Genomics 99(1): 52-58.

McCuddin, Z. P., S. A. Carlson, M. A. Rasmussen and S. K. Franklin (2006). "Klebsiella to Salmonella gene transfer within rumen protozoa: implications for antibiotic resistance and rumen defaunation." Vet Microbiol 114(3-4): 275-284.

Meehan, C. J. and R. G. Beiko (2012). "Lateral gene transfer of an ABC transporter complex between major constituents of the human gut microbiome." $\mathrm{BMC}$ microbiology 12(1): 248.

Minot, S., R. Sinha, J. Chen, H. Li, S. A. Keilbaugh, G. D. Wu, J. D. Lewis and F. D. Bushman (2011). "The human gut virome: inter-individual variation and dynamic response to diet." Genome Res 21(10): 1616-1625.

Moliner, C., P. E. Fournier and D. Raoult (2010). "Genome analysis of microorganisms living in amoebae reveals a melting pot of evolution." FEMS microbiology reviews 34(3): 281-294.

Norman, A., L. H. Hansen and S. J. Sørensen (2009). "Conjugative plasmids: vessels of the communal gene pool." Philosophical Transactions of the Royal Society B: Biological Sciences 364(1527): 2275-2289.

Popa, O., E. Hazkani-Covo, G. Landan, W. Martin and T. Dagan (2011). "Directed networks reveal genomic barriers and DNA repair bypasses to lateral gene transfer among prokaryotes." Genome Res 21(4): 599-609.

Reyes, A., M. Haynes, N. Hanson, F. E. Angly, A. C. Heath, F. Rohwer and J. I. Gordon (2010). "Viruses in the faecal microbiota of monozygotic twins and their mothers." Nature 466(7304): 334-338.

Reyes, A., N. P. Semenkovich, K. Whiteson, F. Rohwer and J. I. Gordon (2012). "Going viral: next-generation sequencing applied to phage populations in the human gut." Nat Rev Microbiol 10(9): 607-617.

Ricard, G., N. R. McEwan, B. E. Dutilh, J. P. Jouany, D. Macheboeuf, M. Mitsumori, F. M. McIntosh, T. Michalowski, T. Nagamine, N. Nelson, C. J. Newbold, E. Nsabimana, A. Takenaka, N. A. Thomas, K. Ushida, J. H. Hackstein and M. A. Huynen (2006). "Horizontal gene transfer from Bacteria to rumen Ciliates indicates adaptation to their anaerobic, carbohydrates-rich environment." BMC Genomics 7: 22.

Ross, E. M., S. Petrovski, P. J. Moate and B. J. Hayes (2013). "Metagenomics of rumen bacteriophage from thirteen lactating dairy cattle." BMC microbiology 13(1): 242. 
Smillie, C. S., M. B. Smith, J. Friedman, O. X. Cordero, L. A. David and E. J. Alm (2011). "Ecology drives a global network of gene exchange connecting the human microbiome." Nature 480(7376): 241-244.

Stecher, B., R. Denzler, L. Maier, F. Bernet, M. J. Sanders, D. J. Pickard, M. Barthel, A. M. Westendorf, K. A. Krogfelt, A. W. Walker, M. Ackermann, U. Dobrindt, N. R. Thomson and W.-D. Hardt (2012). "Gut inflammation can boost horizontal gene transfer between pathogenic and commensal Enterobacteriaceae." Proceedings of the National Academy of Sciences 109(4): 1269-1274.

Thomas, C. M. and K. M. Nielsen (2005). "Mechanisms of, and Barriers to, Horizontal Gene Transfer between Bacteria." Nat Rev Micro 3(9): 711-721.

Zaneveld, J. R., C. Lozupone, J. I. Gordon and R. Knight (2010). "Ribosomal RNA diversity predicts genome diversity in gut bacteria and their relatives." Nucleic Acids Research 38(12): 3869-3879. 